PROCEEDINGS OF THE

AMERICAN MATHEMATICAL SOCIETY

Volume 128, Number 11, Pages 3241-3249

S 0002-9939(00)05623-9

Article electronically published on April 28, 2000

\title{
DISTORTION THEOREMS FOR HIGHER ORDER SCHWARZIAN DERIVATIVES OF UNIVALENT FUNCTIONS
}

\author{
ERIC SCHIPPERS
}

(Communicated by Albert Baernstein II)

\begin{abstract}
Let $\tilde{\mathcal{S}}$ denote the class of functions which are univalent and holomorphic on the unit disc. We derive a simple differential equation for the Loewner flow of the Schwarzian derivative of a given $f \in \tilde{\mathcal{S}}$. This is used to prove bounds on higher order Schwarzian derivatives which are sharp for the Koebe function. As well we prove some two-point distortion theorems for the higher order Schwarzians in terms of the hyperbolic metric.
\end{abstract}

\section{INTRODUCTION}

Let $\tilde{\mathcal{S}}$ denote the class of holomorphic functions $\{f: D \longrightarrow \mathbf{C}, f 1-1\}$. Let

$$
S(f)=\frac{f^{\prime \prime \prime}}{f^{\prime}}-\frac{3}{2}\left(\frac{f^{\prime \prime}}{f^{\prime}}\right)^{2}
$$

denote the Schwarzian derivative, and let $\lambda(z)=\frac{1}{\left(1-|z|^{2}\right)}$ be the hyperbolic line element on the disc. It has long been known that univalence is partly characterized by bounds on the Schwarzian derivative. Nehari [5] proved that $f \in \tilde{\mathcal{S}} \Rightarrow|S(f)| \leq$ $6 \lambda(z)^{2}$, and also proved that if $f$ is holomorphic on $D$, then $|S(f)| \leq 2 \lambda(z)^{2}$ implies that $f$ is one to one. Aharanov and Harmelin [2] studied higher order Schwarzian derivatives $\psi_{n}(f)$ with invariance under composition on the left by Möbius transformations $T, \psi_{n}(T \circ f)=\psi_{n}(f)$, and their relation to univalence. Lavie 3] improved Nehari's sufficient condition in terms of these kinds of invariants. Tamanoi 9 ] considered some of the combinatorial and algebraic aspects.

In this paper a differential equation for the Loewner flow of the Schwarzian derivative of a univalent map is derived. This is used to prove bounds on higher order Schwarzians $\sigma_{n}(f)$ which are invariant under composition on the right with Möbius transformations; these bounds are sharp for suitable transforms of the Koebe function. The proof is of interest for its simplicity; it is very similar to Loewner's proof of the bounds on coefficients of inverse functions [8]. Bertilsson [1] recently derived sharp bounds on another series of Schwarzians with this invariance property as a corollary of estimates on coefficients of negative powers of the derivative, using the method of deBranges. (I would like to thank David Minda for bringing this to

Received by the editors December 14, 1998.

1991 Mathematics Subject Classification. Primary 30C55.

Key words and phrases. Schwarzian derivative, univalent functions, hyperbolic metric.

This paper is part of thesis work at the University of Toronto.

(C)2000 American Mathematical Society 
my attention.) We also derive two-point geometric distortion theorems for univalent functions in terms of higher order Schwarzians, making use of some interesting properties of the extremal functions.

Definition 1. The higher order Schwarzian derivatives are defined inductively as follows. For $f: D \longrightarrow \mathbf{C}$, let

$$
\sigma_{3}(f)=\frac{f^{\prime \prime \prime}}{f^{\prime}}-\frac{3}{2}\left(\frac{f^{\prime \prime}}{f^{\prime}}\right)^{2}
$$

and

$$
\sigma_{n+1}(f)=\sigma_{n}(f)^{\prime}-(n-1) \frac{f^{\prime \prime}}{f^{\prime}} \sigma_{n}(f)
$$

For example,

$$
\begin{gathered}
\sigma_{4}(f)=\frac{f^{\prime \prime \prime \prime}}{f^{\prime}}-6 \frac{f^{\prime \prime \prime} f^{\prime \prime}}{f^{\prime 2}}+6\left(\frac{f^{\prime \prime}}{f^{\prime}}\right)^{3}, \\
\sigma_{5}(f)=\frac{f^{\prime \prime \prime \prime \prime}}{f^{\prime}}-10 \frac{f^{\prime \prime \prime \prime} f^{\prime \prime}}{f^{\prime 2}}-6\left(\frac{f^{\prime \prime \prime}}{f^{\prime}}\right)+48 \frac{f^{\prime \prime \prime} f^{\prime \prime}}{f^{\prime 3}}+36\left(\frac{f^{\prime \prime}}{f^{\prime}}\right)^{4} .
\end{gathered}
$$

It's easy to show by induction that for all Möbius transformations $R$, the following invariance property holds:

$$
\sigma_{n}(f \circ R)=\sigma_{n}(f) \circ R \cdot\left(R^{\prime}\right)^{n-1} .
$$

In particular for disc automorphisms $T$ and affine maps $S$,

$$
\lambda(T(z))^{n-1}\left|\sigma_{n}(S \circ f \circ T)(z)\right|=\lambda^{n-1}(z)\left|\sigma_{n}(f) \circ T(z)\right| .
$$

This invariance is suited to families of functions whose domain is fixed as the disc $D$; for instance for the family $\tilde{\mathcal{S}}$, we have that $\sup _{f \in \tilde{\mathcal{S}}} \lambda(z)^{n-1}\left|\sigma_{n}(f)(z)\right|$ is independent of $z$. Derivatives with this invariance property relate formally via a simple identity to derivatives with invariance under post-composition with Möbius transformations, namely,

$$
\left(\frac{\partial^{n-3}}{\partial z^{n-3}} \sigma_{3}\left(f^{-1}\right)\right) \circ f=\frac{\sigma_{n}(f)}{f^{\prime n-1}} .
$$

It is also worth mentioning that Schwarzians with invariance under pre-composition with Möbius transformations can be written in terms of the derivatives of the hyperbolic metric of the image domain.

In the first section we derive the differential equation for the Schwarzian of the Loewner flow of a univalent function $f$ and use this to prove the bounds on $\left|\sigma_{n}(f)\right|$. In the second section, we derive the geometric two-point distortion theorems directly from some elementary observations about the extremal functions.

\section{A SharP EStimate FOR $\sigma_{n}(f)$}

In this section we prove a differential equation for the Schwarzian derivative as a corollary of the Loewner differential equation. Using this it is possible to prove that

$$
\sup _{f \in \tilde{\mathcal{S}}} \lambda(z)^{n-1}\left|\sigma_{n}(f)(z)\right|=4^{n-3}(n-2) ! 6
$$

This is sharp for a suitable transform of the Koebe function. 
We briefly summarize the Loewner method here (for details and theorems see [6], 7]). Let $\mathcal{S}$ denote the class of functions in $\tilde{\mathcal{S}}$ normalized so that $f(0)=0$ and $f^{\prime}(0)=1$. Every $f \in \mathcal{S}$ can be embedded in a Loewner chain; that is, there is a map $f: D \times \mathbf{R}^{+} \longrightarrow G_{t} \subset \mathbf{C}$ with the following properties:

i) $f_{t}(0)=0, f_{t}^{\prime}(0)=e^{t}$,

ii) Each $f_{t}$ is analytic and univalent on $D$,

iii) $\lim _{t \rightarrow t_{0}} f_{t}=f_{t_{0}}$ locally uniformly,

iv) $G_{s} \subseteq G_{t}$ whenever $s \leq t$,

where the dot denotes differentiation with respect to time and the prime denotes differentiation with respect to $z$. Define the functions $\Phi(\zeta, t)=f^{-1} \circ f_{t}(\zeta)$, with domain $f_{t}^{-1}(f(D))$ for each fixed $t$. For fixed $z$, $\Phi$ is absolutely continuous on compact intervals in $t$, so the time derivative exists almost everywhere. Furthermore, for all $t$ at which it exists, $\lim _{h \rightarrow 0}[\Phi(\zeta, t+h)-\Phi(\zeta, t)] / h=\dot{\Phi}(\zeta, t)$ locally uniformly. Also, $\Phi(\zeta, 0)=\zeta$, and $\lim _{t \rightarrow \infty} \Phi\left(e^{-t} \zeta, t\right) \longrightarrow f^{-1}$ locally uniformly, and $\Phi$ satisfies the Loewner differential equation

$$
\dot{\Phi}(\zeta, t)=\zeta p(\zeta, t) \Phi^{\prime}(\zeta, t)
$$

a.e., where $\operatorname{Re}(p) \geq 0, p(0, t)=1$.

Theorem 1. Let $\Phi$ and $p$ be as above. Then,

i) $\sigma_{3}(\Phi)(\zeta, 0)=0, \lim _{t \rightarrow \infty} \sigma_{3}(f)\left(e^{-t} \Phi\right)(\zeta, t)=\sigma_{3}\left(f^{-1}\right)(\zeta)$ locally uniformly,

ii) $\lim _{t \rightarrow t_{0}} \sigma_{3}(\Phi)(\zeta, t)=\sigma_{3}(\Phi)\left(\zeta, t_{0}\right)$ locally uniformly,

iii) $\lim _{h \rightarrow 0}\left[\sigma_{3}(\Phi)(\zeta, t+h)-\sigma_{3}(\Phi)(\zeta, t)\right] / h$ exists a.e., and where it exists, the limit converges locally uniformly.

iv) $\sigma_{3}\left(\Phi_{t}\right)$ satisfies the differential equation

$$
\sigma_{3} \dot{(\Phi)}=\zeta p \sigma_{3}(\Phi)^{\prime}+\left(2 p+2 \zeta p^{\prime}\right) \sigma_{3}(\Phi)+\left(3 p^{\prime \prime}+\zeta p^{\prime \prime \prime}\right) .
$$

Proof. i) follows from the fact that $\Phi(\zeta)=\zeta$, and $\lim _{t \rightarrow \infty} \Phi\left(e^{-t} \zeta, t\right)=f^{-1}(\zeta)$ locally uniformly. Similarly ii) holds since $\Phi(\zeta, t) \rightarrow \Phi\left(\zeta, t_{0}\right)$ locally uniformly, using Cauchy estimates and the fact that $\Phi_{t}^{\prime}(0) \neq 0$ so that $\Phi_{t}^{\prime}(0)^{-1}$ is uniformly bounded away from zero on compact sets in $\zeta$ and $t$. To show iii), we apply Cauchy estimates to $\left[\Phi^{(j)}(\zeta, t+h)-\Phi^{(j)}(\zeta, h)\right] / h$ for $j=-1,1,2,3$ using the fact that $\lim _{h \rightarrow 0}[\Phi(\zeta, t+h)-\Phi(\zeta, t)] / h$ converges locally uniformly. This also justifies interchanging differentiation with respect to $t$ and $\zeta$, and the following calculations can be made using the differential equation (2) in order to prove iv):

$$
\begin{aligned}
\dot{\Phi}^{\prime} & =\Phi^{\prime}\left(p+\zeta p^{\prime}\right)+\Phi^{\prime \prime} \zeta p \\
\dot{\Phi}^{\prime \prime} & =\Phi^{\prime}\left(2 p^{\prime}+\zeta p^{\prime \prime}\right)+\Phi^{\prime \prime}\left(2 p+2 \zeta p^{\prime}\right)+\Phi^{\prime \prime \prime} \zeta p \\
\dot{\Phi}^{\prime \prime \prime} & =\Phi^{\prime}\left(3 p^{\prime \prime}+\zeta p^{\prime \prime \prime}\right)+\Phi^{\prime \prime}\left(6 p^{\prime}+3 \zeta p^{\prime \prime}\right)+\Phi^{\prime \prime \prime}\left(3 p+3 \zeta p^{\prime}\right)+\Phi^{\prime \prime \prime \prime} \zeta p
\end{aligned}
$$

Also,

$$
\frac{\partial}{\partial t}\left(\frac{\Phi^{\prime \prime \prime}}{\Phi^{\prime}}-\frac{3}{2}\left(\frac{\Phi^{\prime \prime}}{\Phi^{\prime}}\right)^{2}\right)=\frac{\dot{\Phi}^{\prime \prime \prime}}{\Phi^{\prime}}-\frac{\Phi^{\prime \prime \prime}}{\Phi^{\prime}} \frac{\dot{\Phi}^{\prime}}{\Phi^{\prime}}-3 \frac{\Phi^{\prime \prime}}{\Phi^{\prime}} \frac{\dot{\Phi}^{\prime \prime}}{\Phi^{\prime}}+3\left(\frac{\Phi^{\prime \prime}}{\Phi^{\prime}}\right)^{2} \frac{\dot{\Phi}^{\prime}}{\Phi^{\prime}} .
$$


Plugging 3 into 4

$$
\begin{aligned}
\left.\sigma_{3} \dot{(} \Phi\right)= & \zeta p \frac{\Phi^{\prime \prime \prime \prime}}{\Phi^{\prime}}+\left(3 p+3 \zeta p^{\prime}\right) \frac{\Phi^{\prime \prime \prime}}{\Phi^{\prime}}+\left(6 p^{\prime}+3 \zeta p^{\prime \prime}\right) \frac{\Phi^{\prime \prime}}{\Phi^{\prime}}+\left(3 p^{\prime \prime}+\zeta p^{\prime \prime \prime}\right) \\
& -\frac{\Phi^{\prime \prime \prime}}{\Phi^{\prime}}\left(\left(p+\zeta p^{\prime}\right)+\zeta p \frac{\Phi^{\prime \prime}}{\Phi^{\prime}}\right) \\
& -3 \frac{\Phi^{\prime \prime}}{\Phi^{\prime}}\left(\left(2 p^{\prime}+\zeta p^{\prime \prime}\right)+\left(2 p+2 \zeta p^{\prime}\right) \frac{\Phi^{\prime \prime}}{\Phi^{\prime}}+\zeta p \frac{\Phi^{\prime \prime \prime}}{\Phi^{\prime}}\right) \\
& +3\left(\frac{\Phi^{\prime \prime}}{\Phi^{\prime}}\right)^{2}\left(\left(p+\zeta p^{\prime}\right)+\zeta p \frac{\Phi^{\prime \prime}}{\Phi^{\prime}}\right) \\
= & \zeta p\left(\frac{\Phi^{\prime \prime \prime \prime}}{\Phi^{\prime}}-4 \frac{\Phi^{\prime \prime \prime} \Phi^{\prime \prime}}{\Phi^{\prime 2}}+3\left(\frac{\Phi^{\prime \prime}}{\Phi^{\prime}}\right)^{3}\right) \\
& +\left(2 p+2 \zeta p^{\prime}\right)\left(\frac{\Phi^{\prime \prime \prime}}{\Phi^{\prime}}-\frac{3}{2}\left(\frac{\Phi^{\prime \prime}}{\Phi^{\prime}}\right)^{2}\right)+\left(3 p^{\prime \prime}+\zeta p^{\prime \prime \prime}\right) .
\end{aligned}
$$

In order to prove the bounds on $\sigma_{n}(f)$, we will need Caratheodory's lemma, which states that the coefficients $c_{n}$ of an analytic function $p$ on the unit disc with $p(0)=1$ and $\operatorname{Re}(p(z)) \geq 0$ satisfy $\left|c_{n}\right| \leq 2, n \geq 2$. For all $n$, equality $\left|c_{1}\right|=\cdots=\left|c_{n}\right|=2$ holds iff $p$ is given by

$$
p(z)=\frac{1+e^{i \theta} z}{1-e^{i \theta} z}=1+2 e^{i \theta} z+2 e^{2 i \theta} z^{2}+\cdots .
$$

If we let $p(z, t)$ be one of the extremal functions given above, then the solution $\Phi$ of the differential equation (2) generates the inverse of a rotation of the Koebe function $\tilde{k}(z)=\frac{z}{\left(1-e^{i \theta} z\right)^{2}}$ as $t \rightarrow \infty($ see [8] $)$.

\section{Theorem 2.}

$$
\sup _{f \in \tilde{\mathcal{S}}} \lambda(z)^{n-1}\left|\sigma_{n}(f)(z)\right|=4^{n-3}(n-2) ! 6 .
$$

This is sharp iff $f=S \circ k \circ T$ where $k$ is the Koebe function, $S$ an affine transformation, and $T$ a disc automorphism.

Proof. Using the invariances of $\sigma_{n}(f)$, it suffices to prove this at the origin for functions with $f(0)=0$ and $f^{\prime}(0)=1$. Let $\Phi$ be the transition function for a Loewner chain starting at $f$, and let $p$ be the infinitesimal generator, as above. Now

$$
\sigma_{3}(\Phi)=s_{0}(t)+s_{1}(t) \zeta+s_{2}(t) \zeta^{2}+\cdots
$$

where

$$
s_{n}(t)=\frac{1}{n !} \frac{\partial^{n}}{\partial z^{n}} \sigma_{3}(\Phi)(0)
$$

By Theorem 1,

$$
\lim _{t \rightarrow \infty} e^{-(n+2) t} s_{n}(t)=\frac{1}{n !}\left(\frac{\partial^{n}}{\partial z^{n}} \sigma_{3}\left(f^{-1}\right)\right)(0) .
$$


By (1), and the normalization $f^{\prime}(0)=1$,

$$
\lim _{t \rightarrow \infty} e^{-(n+2) t} s_{n}(t)=\frac{1}{n !} \sigma_{n+3}(f)(0) .
$$

We can expand the differential equation of Theorem 1 in a power series and equate coefficients. Suppressing $t$,

$$
\begin{gathered}
\left.\sigma_{3} \dot{(} \Phi\right)=\dot{s}_{0}+\dot{s}_{1} \zeta+\dot{s}_{2} \zeta^{2}+\cdots \\
\zeta p \sigma_{3}(\Phi)^{\prime}=\zeta\left(\sum_{n=0}^{\infty} c_{n} \zeta^{n}\right)\left(\sum_{n=0}^{\infty}(n+1) s_{n+1} \zeta^{n}\right) \\
=\sum_{n=0}^{\infty}\left(\sum_{k=0}^{n} c_{n-k}(k+1) s_{k+1}\right) \zeta^{n+1}
\end{gathered}
$$

Also,

So

$$
\left(2 p+2 \zeta p^{\prime}\right)=2\left(\sum_{n=0}^{\infty} c_{n} \zeta^{n}+\sum_{n=1}^{\infty} n c_{n} \zeta^{n}\right)=2\left(\sum_{n=0}^{\infty}(n+1) c_{n} \zeta^{n}\right) .
$$

$$
\begin{aligned}
\left(2 p+2 \zeta p^{\prime}\right) \sigma_{3}(\Phi) & =2\left(\sum_{n=0}^{\infty}(n+1) c_{n} \zeta^{n}\right)\left(\sum_{n=0}^{\infty} s_{n} \zeta^{n}\right) \\
& =2 \sum_{n=0}^{\infty}\left(\sum_{k=0}^{n}(n+1-k) c_{n-k} s_{k}\right) \zeta^{n} \\
3 p^{\prime \prime}+\zeta p^{\prime \prime \prime} & =3 \sum_{n=2}^{\infty} n(n-1) c_{n} \zeta^{n-2}+\zeta \sum_{n=3}^{\infty} n(n-1)(n-2) c_{n} \zeta^{n-3} \\
& =\sum_{n=2}^{\infty}(n+1) n(n-1) c_{n} \zeta^{n-2} \\
& =\sum_{n=0}^{\infty} \frac{(n+3) !}{n !} c_{n+2} \zeta^{n} .
\end{aligned}
$$

So applying Theorem [1 to the expansion (5) using (7), (8), (9), and (10), and equating coefficients, we get the following simple recursive differential equation for $s_{n}$ :

$$
\dot{s}_{n}=\sum_{k=0}^{n}(2(n+1)-k) c_{n-k} s_{k}+\frac{(n+3) !}{n !} c_{n+2}
$$

or, bringing the $k=0$ term to the left hand side,

$$
\frac{d}{d t}\left(e^{-(n+2) t} s_{n}\right)=e^{-(n+2) t}\left(\sum_{k=1}^{n}(2 n+2-k) c_{n-k} s_{k}+\frac{(n+3) !}{n !} c_{n+2}\right) .
$$

So (compare Schober [8])

$$
e^{-(n+2) t} s_{n}=\int_{0}^{t} e^{-(n+2) \tau}\left(\sum_{k=1}^{n}(2 n+2-k) c_{n-k}(\tau) s_{k}(\tau)+\frac{(n+3) !}{n !} c_{n+2}(\tau)\right) d \tau
$$


By Caratheodory's lemma, each $c_{k}$ is bounded in modulus by 2 . So the maximum of the right hand side can occur for each fixed $t$ iff $\left|c_{1}\right|=\cdots\left|c_{n}\right|=\left|c_{n+2}\right|$. By the discussion preceding the theorem, then, $p(z, t)=\frac{1+2 e^{i \theta}}{1-2 e^{i \theta}}$ for all $t$ (it's not hard to check that the maximum is indeed attained for all of these). Thus,

$$
\left|\sigma_{n}(f)(0)\right|=\left|\lim _{t \rightarrow \infty} e^{-(n+2) t} s_{n}(t)\right| \leq\left|\sigma_{n}(\tilde{k})(0)\right|
$$

where $\tilde{k}$ is a rotation of the Koebe function. A calculation shows that $\left|\sigma_{n}(\tilde{k})(0)\right|=$ $4^{n-3}(n-2) ! 6$.

\section{Geometric two-Point Distortion theOREMS FOR THE CLASS $\tilde{\mathcal{S}}$}

We now derive a two-point distortion theorem for the higher order Schwarzians. First it is necessary to derive a two-point geometric version of the classical distortion theorem for the class $\mathcal{S}$. Though easy to prove, it is of interest in its own right. It is similar to a two-point reformulation of the classical growth theorem due to Kim and Minda 4] (which is in fact also sufficient for univalence).

After Minda and Peschl, denote

$$
D_{i} f(a)=\left.\frac{\partial^{i}}{\partial z^{i}}\right|_{z=0} f \circ T_{a}
$$

where $T_{a}(z)=\frac{z+a}{1+\bar{a} z}$. In particular for $i=1,2$,

$$
\begin{aligned}
& D_{1} f(z)=\left(1-|z|^{2}\right) f^{\prime}(z) \\
& D_{2} f(z)=\left(1-|z|^{2}\right)^{2} f^{\prime \prime}(z)+2 \bar{z}\left(1-|z|^{2}\right) f^{\prime}(z)
\end{aligned}
$$

These satisfy the invariance

$$
D_{n}(f \circ T)=D_{n}(f) \circ T \cdot \frac{T^{\prime n}}{\left|T^{\prime n}\right|} .
$$

Theorem 3. If $f \in \tilde{\mathcal{S}}$, then $\forall z_{1}, z_{2} \in D$,

$$
\exp \left(-4 d_{\lambda}\left(z_{1}, z_{2}\right)\right) \leq \frac{\left|D_{1} f\left(z_{2}\right)\right|}{\left|D_{1} f\left(z_{1}\right)\right|} \leq \exp \left(4 d_{\lambda}\left(z_{1}, z_{2}\right)\right)
$$

where $d_{\lambda}\left(z_{1}, z_{2}\right)$ is the hyperbolic distance between $z_{1}$ and $z_{2}$. This is sharp in the following way: fix $z_{1}$, and let $z_{2}$ vary along a hyperbolic geodesic through $z_{1}$. If $T$ is any disc automorphism taking this geodesic to the real axis, $S$ any affine transformation of the plane, and $k$ the Koebe function, then the function $\tilde{k}=S \circ k \circ T$ takes on the upper bound if $T\left(z_{2}\right) \geq T\left(z_{1}\right)$, and the lower bound if $T\left(z_{1}\right) \geq T\left(z_{2}\right)$. Furthermore for this function, arg $\frac{D_{1} \tilde{k}\left(z_{2}\right)}{D_{1} \tilde{k}\left(z_{1}\right)}$ is constant while $z_{2}$ remains on one side of $z_{1}$.

Proof. Let $T_{a}(z)=\frac{z+a}{1+\bar{a} z}$. Given $f \in \tilde{\mathcal{S}}$, the function

$$
g(z)=\frac{f \circ T_{a}(z)-f \circ T_{a}(0)}{\left(f \circ T_{a}\right)^{\prime}(0)}
$$


is in $\mathcal{S}$, so the second coefficient of $g$, namely $\frac{1}{2} \frac{D_{2} f(z)}{D_{1} f(z)}$, is bounded in modulus by 2. Thus $\forall f \in \tilde{\mathcal{S}},\left|\frac{D_{2} f(z)}{D_{1} f(z)}\right| \leq 4$. Now by an easy computation

$$
d \log \left|D_{1} f\right|=R e\left(\lambda \frac{D_{2} f(z)}{D_{1} f(z)} d z\right) .
$$

So for all smooth curves $\gamma$ joining $z_{1}$ to $z_{2}$,

$$
\log \left|D_{1} f\left(z_{2}\right)\right|-\log \left|D_{1} f\left(z_{1}\right)\right|=\int_{\gamma} \operatorname{Re}\left(\lambda \frac{D_{2} f(z)}{D_{1} f(z)} d z\right) .
$$

Now for the Koebe function $k, \frac{D_{2} k(z)}{D_{1} k(z)}=4 \forall z \in(-1,1)$. Let $\alpha$ be the geodesic segment joining $z_{1}$ to $z_{2}$ (parametrized with respect to arc length, increasing from $z_{1}$ to $\left.z_{2}\right)$. Let $T$ be a disc automorphism taking $\alpha$ to the real line, with $T\left(z_{2}\right) \geq T\left(z_{1}\right)$. Then $k \circ T$ satisfies (using (17))

$$
\begin{aligned}
\operatorname{Re}\left(\lambda \circ \alpha \frac{D_{2}(k \circ T) \circ \alpha}{D_{1}(k \circ T) \circ \alpha} \alpha^{\prime}\right) & =\operatorname{Re}\left(\lambda \circ \alpha \frac{\left(D_{2} k\right) \circ T \circ \alpha}{\left(D_{1} k\right) \circ T \circ \alpha} \frac{T^{\prime} \circ \alpha}{\left|T^{\prime} \circ \alpha\right|} \alpha^{\prime}\right) \\
& =\operatorname{Re}\left(\lambda \circ(T \circ \alpha) \frac{D_{2}(k \circ T) \circ \alpha}{D_{1}(k \circ T) \circ \alpha}(T \circ \alpha)^{\prime}\right) \\
& =4 .
\end{aligned}
$$

Similarly, if we choose $T$ such that $T\left(z_{2}\right) \leq T\left(z_{1}\right)$, we get that

$$
\operatorname{Re}\left(\lambda \circ \alpha \frac{D_{2}(k \circ T) \circ \alpha}{D_{1}(k \circ T) \circ \alpha} \alpha^{\prime}\right)=-4 .
$$

So when the lower and upper bound of the right hand side of (18) are taken on, it is along the entire geodesic segment. Since the integral of $d \log \left|D_{1} f\right|$ is independent of path, we can fix the path as a hyperbolic geodesic $\alpha$ connecting $z_{1}$ to $z_{2}$. We get that the maximum and minimum of the integral (18) must be taken on by these functions, so

$$
-\int_{\alpha} 4 \lambda|d z| \leq \log \frac{\left|D_{1} f\left(z_{2}\right)\right|}{\left|D_{1} f\left(z_{1}\right)\right|} \leq \int_{\alpha} 4 \lambda|d z|
$$

which implies that

$$
-4 d_{\lambda}\left(z_{1}, z_{2}\right) \leq \log \frac{\left|D_{1} f\left(z_{2}\right)\right|}{\left|D_{1} f\left(z_{1}\right)\right|} \leq 4 d_{\lambda}\left(z_{1}, z_{2}\right) .
$$

That these are all the sharp functions follows directly from the fact that the only functions in $\mathcal{S}$ with $\left|a_{2}\right|=2$ are the Koebe function and its rotations.

We now use this to get a two-point distortion theorem involving $\sigma_{n}(f)$, also in terms of hyperbolic distance.

Theorem 4. Let $c_{n}=4^{n-3}(n-2) ! 6$. The estimate

$$
\left|\frac{f^{\prime}\left(z_{1}\right)^{n-1}}{f^{\prime}\left(z_{2}\right)^{n-1}} \sigma_{n}(f)\left(z_{2}\right)-\sigma_{n}(f)\left(z_{1}\right)\right| \leq c_{n} \lambda\left(z_{1}\right)^{n-1} \exp \left(4(n-1) d_{\lambda}\left(z_{1}, z_{2}\right)\right)
$$

holds $\forall f \in \tilde{\mathcal{S}}$, and this is sharp only for $f=S \circ k \circ T$. 
Proof. We have

$$
\frac{\partial}{\partial z}\left(\frac{\sigma_{n}(f)}{f^{\prime n-1}}\right)=\frac{\sigma_{n+1}(f)}{f^{\prime n-1}}
$$

so

$$
\left|\frac{f^{\prime}\left(z_{1}\right)^{n-1}}{f^{\prime}\left(z_{2}\right)^{n-1}} \sigma_{n}(f)\left(z_{2}\right)-\sigma_{n}(f)\left(z_{1}\right)\right|=\left|\int_{z_{1}}^{z_{2}} \frac{f^{\prime}\left(z_{1}\right)^{n-1}}{f^{\prime}(z)^{n-1}} \sigma_{n+1}(f)(z) d z\right| .
$$

Assume $\left|\sigma_{n+1}(f)(z)\right| \leq c_{n+1} \lambda(z)^{n} \forall f \in \tilde{\mathcal{S}}$. First we observe that it suffices to estimate the right hand side fixing the path of integration as a hyperbolic geodesic. Denote this again by $\alpha$, travelling from $z_{1}$ to $z_{2}$. Next, using

$$
\sigma_{n}(k)(z)=\frac{(-1)^{n} c_{n}}{\left(1-z^{2}\right)^{n-1}}
$$

an argument similar to that in the last theorem shows that for a disc automorphism $T$ taking $\alpha$ to the real axis, the function $\tilde{k}=k \circ T$ satisfies

$$
\left|\sigma_{n+1}(\tilde{k})\right| \circ \alpha=c_{n+1}(\lambda \circ \alpha)^{n}
$$

and along $\alpha$,

$$
\arg \left(\frac{\tilde{k}^{\prime}\left(z_{1}\right)^{n-1}}{\tilde{k}^{\prime}(z)^{n-1}} \sigma_{n+1}(\tilde{k})(z) d z\right)
$$

is constant. Applying Theorem [3, we get

$$
\begin{aligned}
\left|\int_{\alpha} \frac{f^{\prime}\left(z_{1}\right)^{n-1}}{f^{\prime}(z)^{n-1}} \sigma_{n+1}(f)(z) d z\right| & \leq \int_{\alpha} \exp \left(4(n-1) d_{\lambda}\left(z_{1}, z\right)\right) \frac{\lambda\left(z_{1}\right)^{n-1}}{\lambda(z)^{n-1}} c_{n+1} \lambda(z)^{n}|d z| \\
& =\lambda\left(z_{1}\right)^{n-1} \int_{\alpha} \exp \left(4(n-1) d_{\lambda}\left(z_{1}, z\right)\right) c_{n+1} \lambda(z)|d z| .
\end{aligned}
$$

To integrate the right hand side, let

$$
x(t)=\left|\frac{\alpha(t)-z_{1}}{1-\overline{z_{1}} \alpha(t)}\right|=T \circ \alpha(t)
$$

where $T$ is the disc automorphism above, and note that

$$
\dot{x}(t)=T^{\prime}(\alpha(t)) \dot{\alpha}(t)=\left|T^{\prime}(\alpha(t))\right||\dot{\alpha}(t)| .
$$

Also let $u(x)=\frac{1}{2} \log \frac{1+x}{1-x}$. So $u(x(t))=d_{\lambda}\left(\alpha(t), z_{1}\right)$ and

$$
\begin{aligned}
\frac{d u}{d t} & =\frac{1}{1-x^{2}} T^{\prime}(\alpha(t)) \dot{\alpha}(t)=\frac{|\alpha \dot{(t)}|}{1-|\alpha(t)|^{2}} \\
& =\lambda(\alpha(t))|\alpha(t)| .
\end{aligned}
$$

Thus,

$$
\begin{aligned}
\int_{\alpha} \exp 4(n-1) d_{\lambda}\left(z, z_{1}\right) c_{n+1} \lambda(z)|d z| \\
\quad=c_{n+1} \int_{0}^{\alpha^{-1}\left(z_{2}\right)} \exp 4(n-1) u \circ x(t) d(u \circ x) \\
\quad=c_{n} \exp 4(n-1) d_{\lambda}\left(z_{1}, z_{2}\right) .
\end{aligned}
$$

It's easy to see that this theorem also implies Theorem 2 . 


\section{REFERENCES}

[1] Bertilsson, Daniel. Coefficient estimates for negative powers of the derivative of univalent functions. Ark. Mat. 36 (1998), no. 2, 255-273. MR 99i:30030

[2] Harmelin, Reuven. Aharanov invariants and univalent functions. Israel J. Math 43 (1982) 3 pp 244-254. MR 85a:30020

[3] Lavie, Meira. The Schwarzian derivative and disconjugacy of nth order linear differential equations. Canad. J. Math. 21 (1969).

[4] Kim, Seong-A and Minda, David. Two point distortion theorems for univalent functions. Pacific J. of Math. 1631 pp 137-157. MR 94m:30042

[5] Nehari, Zeev. The Schwarzian derivative and schlicht functions. Bull. Amer. Math. Soc. 55 (1949) pp 545-551. MR 10:696e

[6] Pommerenke, Christian. Univalent Functions. Vandenhoeck and Ruprecht, Göttingen 1975. MR 58:22526

[7] Rosenblum, Marvin and Rovnyak, James. Topics in Hardy Classes and Univalent Functions. Birkhäuser Advanced Texts, 1994. MR 97a:30047

[8] Schober, Glenn. Coefficient estimates for inverses of schlicht functions. In Aspects of Contemporary Complex Analysis, ed. Brannan, D.A. and Clunie, J.G. 1980, pp. 503-513. MR 82j:30021

[9] Tamanoi, Hirotaka. Higher Schwarzian operators and combinatorics of the Schwarzian derivative. Math. Ann. 305 (1996) 1 pp 127-151. MR 97h:30001

Department of Mathematics, University of Toronto, 100 St. George St., Toronto, Ontario, Canada M5S 3G3

E-mail address: eric@math.toronto.edu 